\title{
Phosphate control of phoA, phoC and phoD gene expression in Streptomyces coelicolor reveals significant differences in binding of PhoP to their promoter regions \\ Correspondence \\ Juan Francisco Martín \\ jf.martin@unileon.es \\ Received 15 February 2007 \\ Revised 21 June 2007 \\ Accepted 26 June 2007

\author{
Alexander K. Apel, ${ }^{1,2}$ Alberto Sola-Landa, ${ }^{1}$ Antonio Rodríguez-García ${ }^{1}$ \\ and Juan F. Martín ${ }^{1,2}$ Spain
${ }^{2}$ Área de Microbiología, Fac. CC. Biológicas y Ambientales, Universidad de León, Campus de Vegazana, s/n, 24071, León, Spain \\ ${ }^{1}$ Instituto de Biotecnología de León, INBIOTEC, Parque Científico de León, Av. Real 1, 24006 León,
}

\begin{abstract}
Three putative alkaline phosphatase genes, pho $A, p h o C$ and $p h o D$, were identified in the genome of Streptomyces coelicolor by homology with the amino acid sequence obtained from the PhoA protein of Streptomyces griseus. PhoA and PhoC correspond to broad-spectrum alkaline phosphatases whereas $\mathrm{PhoD}$ is similar to a $\mathrm{Ca}^{2+}$-dependent phospholipase $\mathrm{D}$ of Streptomyces chromofuscus. The phoA and phoD genes were efficiently expressed in R5 medium under phosphate-limited conditions, as shown by studies using the $x y l E$ reporter gene, whereas phoC was poorly transcribed under the same conditions. Expression of phoA was clearly PhoPdependent since it was not transcribed in the $S$. coelicolor $\Delta p h o P$ mutant and was strongly activated under low phosphate concentrations. Similarly, expression of phoD was PhoPdependent and highly sensitive to phosphate availability. By contrast, expression of phoC was not PhoP-dependent. Electrophoretic mobility shift assays showed that PhoP binds to the phoA and phoD promoters, but not to that of phoC. Footprinting studies with GST-PhoP revealed the presence of a $\mathrm{PHO}$ box (two direct 11 nt repeats) in the phoA promoter and two $\mathrm{PHO}$ boxes in the promoter of $p h o D$. The transcription start points of the three promoters were identified by primer extension. The transcription start point of $p h o D$ coincides with the $\mathrm{G}$ of its translation start codon, indicating that this gene is transcribed as a leaderless mRNA. The deduced -10 and -35 regions of $p h o D$ (but not those of $p h o A$ ) overlapped with the $\mathrm{PHO}$ boxes in this promoter, suggesting that an excess of PhoP interferes with binding of the RNA polymerase to this promoter. In summary, the three promoters showed clear differences in the modulation of their expression by PhoP.
\end{abstract}

\section{INTRODUCTION}

Streptomyces species are soil dwelling micro-organisms that secrete many extracellular enzymes to degrade polymeric substrates and to utilize the nutrients obtained after their hydrolysis (García-González et al., 1991; Fernández-Ábalos et al., 1992; Schaerlaekens et al., 2004). Phosphate is an essential nutrient for growth of all micro-organisms. Streptomyces species and other soil micro-organisms may use inorganic phosphate, which occurs frequently in the form of partially insoluble phosphate salts, and organic phosphate, which is present in a variety of plant materials such as phytic acid (Martínez-Domínguez et al., 2002), sugar phosphates and nucleotides (Martín \& Demain,

Abbreviations: EMSA, electrophoretic mobility shift assay; DBD, DNAbinding domain; GST, glutathione S-transferase.
1977). To obtain phosphate from organic compounds, Streptomyces and other bacteria use an extracellular alkaline phosphatase encoded by the phoA gene (Moura et al., 2001). When the Streptomyces griseus phoA gene was cloned and compared with the genome of Streptomyces coelicolor, two other putative phosphatase genes (hereafter named phoC and phoD; $p h o B$ was avoided because this designation is used for the response regulator in Escherichia coli), in addition to the $p h o A$ orthologue, were found (Moura et al., 2001; Bentley et al., 2002). Different phosphatases may work on different phosphorylated substrates.

Species of the genus Streptomyces are the major producers of secondary metabolites, including antibiotics and other pharmacologically active compounds, pigments, toxins, plant growth factors, etc. (von Döhren \& Gräfe, 1997; Demain \& Fang, 2000; Martín et al., 2000). The 
biosynthesis of most of these secondary metabolites is negatively controlled by phosphate and their production occurs only under phosphate-limited conditions (Martín \& Demain, 1980; Martín et al., 1994).

Expression of phosphate-regulated genes is modulated by the two-component system PhoR-PhoP (Sola-Landa et al., 2003; Ghorbel et al., 2006). Binding of the responseregulator PhoP to the promoters of phosphate-regulated genes was shown to modulate expression of primary metabolism genes, including phoA, by Western blot analysis (Sola-Landa et al., 2003), and genes involved in actinorhodin and undecylprodigiosin biosynthesis. Binding of the response regulator PhoP to the promoter regions of three genes of the pho regulon, pstS, phoU and phoRP, was shown both in S. coelicolor (Sola-Landa et al., 2005) and Streptomyces natalensis (Mendes et al., 2007), but direct binding of PhoP to the promoters of secondary metabolite biosynthesis genes has not been reported (Martín, 2004).

Synthesis of the PhoA alkaline phosphatase, as shown by Western blot analysis, is regulated positively by PhoP since in deletion mutants (either $\triangle p h o P$ or $\triangle p h o R P$ ) this protein is not detected (Sola-Landa et al., 2003). However, it is not known whether the two-component system exerts its action by direct binding of PhoP to specific sequences in the $p h o A$ promoter region or whether regulation proceeds through other DNA-binding proteins by a cascade mechanism. Moreover, it is not known if the different phosphatase genes are regulated or not by phosphate availability or whether they respond to the same or to different type(s) of phosphate control.

It was, therefore, important to perform a comparative study of the mechanism of phosphate control of phoA,
phoC and phoD expression using DNA electrophoretic mobility shift assays (EMSAs) as well as reporter gene expression.

In this article we report significant differences in the control of the three putative phosphatase-encoding genes. EMSA and footprinting analyses provided evidence for the presence of PhoP-binding sequences (PHO boxes) in the promoters of phoA and phoD but not in that of phoC, which is regulated by a different mechanism.

\section{METHODS}

Bacterial strains and plasmids. The bacterial strains and plasmids used in this work are listed in Table 1. Oligonucleotides were designed to amplify $500 \mathrm{bp}$ fragments of the promoters of phoA, phoC and phoD containing sites for the restriction enzymes HindIII in the forward (F) and BglII or BamHI in the reverse (R) primers (underlined in the sequences below).

The phoA promoter was cloned from cosmid SCC75A by PCR with the primer pair F1-TAATAAGCTTCCCGTTGAGGTTGGTGTC and R1-ATAGATCTGTTCCTCCCCTTGCGG, resulting in a $517 \mathrm{bp}$ fragment containing the promoter and the first two bases of the coding sequence. The phoC promoter was cloned from cosmid SCF43A by PCR with the primer pair F2-TAATAAGCTTCACCGCTGCCGTCGTA and R2-TGGGATCCATCTGGTGTCCTCTCGTA, resulting in a $532 \mathrm{bp}$ fragment containing the promoter and the first 10 bases of the coding sequence. The phoD promoter was cloned from cosmid SC4G6 by PCR with the primer pair F3-TAATAAGCTTCAAGACCGCGCTGTACTCCACGAA and R3-TAGGATCDAGCGGGGCGGCGAGCAC, resulting in a 529 bp fragment containing the promoter and the first 98 bases of the coding sequence (a region important for expression of this gene; see Results). The different nucleotide distance from the fusion site to the translation start codon of the $x y l E$ gene for the phoD promoter as compared to those of $p h o A$ and phoC might affect the intensity of expression from the different promoters.

Table 1. Bacterial strains and plasmids

\begin{tabular}{|c|c|c|}
\hline Strain/plasmid & Characteristics & Reference \\
\hline \multicolumn{3}{|l|}{ Strains } \\
\hline S. lividans JI 1326 & Wild-type & John Innes Centre \\
\hline S. coelicolor M145 & Wild-type & Kieser et al. (2000) \\
\hline S. coelicolor INB101 & $S$ coelicolor M145 $\Delta p h o P$ & Rodríguez-García et al. (2007) \\
\hline E. coli $\mathrm{DH} 5 \alpha$ & $\begin{array}{l}\mathrm{F}^{\prime} \phi 80 \mathrm{~d} \text { lacZ } \triangle \mathrm{M} 15 \Delta(\text { lacZYA-argF }) \text { U16 recA1 endA1 hsdR17 } \\
\left(\mathrm{r}^{-} \mathrm{m}^{+}\right) \text {supE44 med } \lambda^{-} \text {thi-1 gyrA relA1 }\end{array}$ & Hanahan (1983) \\
\hline \multicolumn{3}{|c|}{ 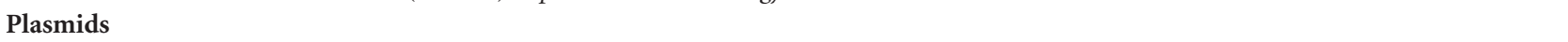 } \\
\hline pGEM-T Easy & Vector system for the cloning of PCR products & Promega \\
\hline pGEM-PphoA & PCR product carrying the $p h o A$ promoter cloned into pGEM-T Easy & This work \\
\hline pGEM-P phoC & PCR product carrying the $p h o C$ promoter cloned into pGEM-T Easy & This work \\
\hline pGEM-PphoD & PCR product carrying the $p h o D$ promoter cloned into pGEM-T Easy & This work \\
\hline pGEM-P phoAp & pGEM-PphoA minus the MluI-BamHI fragment & This work \\
\hline pGEM-P phoDp & pGEM-P phoD minus the $M l u \mathrm{I}-M l u \mathrm{I}$ fragment & This work \\
\hline pIJ4083 & Promoter-probe plasmid using $x y l E$ as reporter & Clayton \& Bibb (1990) \\
\hline pIJ4083-PphoA & phoA promoter cloned into pIJ4083 & This work \\
\hline pIJ4083-PphoC & phoC promoter cloned into pIJ4083 & This work \\
\hline pIJ4083-PphoD & phoD promoter cloned into pIJ4083 & This work \\
\hline
\end{tabular}

${ }^{*}$ Collection of micro-organisms of the John Innes Centre, Colney Lane, Norwich NR4 7UH, UK. 
After PCR amplification the promoters were cloned into pGEM-T Easy (Promega). All the promoters were sequenced using pUC/M13 forward and reverse primers in a Perkin Elmer ABI PRISM 310 Genetic Analyzer, extracted from the plasmid via digestion with HindIII/BglII or HindIII/BamHI and ligated into pIJ4083 in order to construct transcriptional fusions with the $x y l E$ reporter gene (Clayton \& Bibb, 1990). The obtained promoter-probe plasmids pIJ4083PphoA, pIJ4083-PphoC and pIJ4083-PphoD were first transformed into Streptomyces lividans, then extracted, confirmed by restriction with HindIII and XbaI and transformed into S. coelicolor.

Promoter expression studies. Promoter expression was studied using the $x y l E$ reporter gene of plasmid pIJ4083. Approximately $3 \times 10^{6}$ spores were pregerminated in $2 \times$ TY medium (Kieser et al., 2000) for $8 \mathrm{~h}$ at $30{ }^{\circ} \mathrm{C}$ and 250 r.p.m. After centrifugation they were resuspended in $100 \mu \mathrm{l}$ R5 medium (Kieser et al., 2000) and used to inoculate $100 \mathrm{ml}$ R5 medium supplemented with high $(1.8 \mathrm{mM}$ $\left.\mathrm{KH}_{2} \mathrm{PO}_{4}\right)$ or low $\left(0.009 \mathrm{mM} \mathrm{KH_{2 }} \mathrm{PO}_{4}\right)$ concentrations of added phosphate. Thiostrepton was used as selective antibiotic at a final concentration of $5 \mu \mathrm{g} \mathrm{ml}^{-1}$. Samples were taken from two flasks at $18,24,27,30,33,36,39$ and $48 \mathrm{~h}$ and catechol-2,3-dioxygenase activity was measured in triplicate as described by Kieser et al. (2000).

DNA-protein binding assays. To detect binding of PhoP to the promoters, studies were performed by EMSAs using glutathione $S$ transferase (GST)-PhoP and GST-PhoP ${ }^{\mathrm{DBD}}$ (DNA-binding domain) fusion proteins as described by Sola-Landa et al. (2005). The promoters were excised from the pGEM-T Easy plasmid with restriction enzymes and labelled at both ends with digoxigenin using the DIG Oligonucleotide $3^{\prime}$-Labelling kit, 2nd generation (Roche Applied Science).

Samples were loaded onto a $5 \%$ polyacrylamide native gel $(29: 1)$ in $0.5 \%$ TBE buffer and electrophoresis was run for $5 \mathrm{~h}$ at $80 \mathrm{~V}$. Afterwards the gel was electroblotted onto a nylon membrane for $1 \mathrm{~h}$ at $200 \mathrm{~mA}$ in $0.5 \%$ TBE buffer and the DNA was fixed by UV crosslinking to the membrane. Labelled DNA was detected with antidigoxigenin antibodies by chemoluminiscence development with CDP-Star reagent (Roche Applied Science).

Footprinting assays. To obtain small promoter fragments, needed for footprinting studies, a fragment was deleted from the plasmids by digestion with BamHI/MluI in the case of pGEM-PphoA and with MluI alone in the case of pGEM-P phoD, using restriction sites located in the promoter and the flanking plasmid region but maintaining the annealing sequences for M13-20 and reverse primers. The plasmids obtained by religation were used to amplify the promoter fragments of phoA (P phoAp) and phoD (PphoDp) for DNAse I footprinting studies using M13-20 and reverse primers, one of them fluorescein-labelled. The assays were performed by the fluorescein labelling procedure as described by Rodríguez-García et al. (1997) using the GST-PhoP ${ }^{\mathrm{DBD}}$ protein as described by Sola-Landa et al. (2005). After amplification the PCR products were purified by agarose-gel electrophoresis and the DNA concentrations were determined by spectrophotometry with a GeneQuant spectrophotometer (Amersham Biosciences).

The reaction components were the same as in the DNA binding studies. Labelled DNA fragments $(0.28 \mathrm{pmol})$ and GST-PhoP ${ }^{\mathrm{DBD}}$ protein were added to a final volume of $28 \mu \mathrm{l}$, and incubated at $30{ }^{\circ} \mathrm{C}$ for $30 \mathrm{~min}$. Lyophilized bovine pancreas DNase I (Roche grade I) was reconstituted in $20 \mathrm{mM}$ Tris/ $\mathrm{HCl}, \mathrm{pH} 7.0,50 \mathrm{mM} \mathrm{NaCl}$, $100 \mu \mathrm{g} \mathrm{ml}^{-1}$ BSA, $1 \mathrm{mM}$ DTT and $50 \%$ glycerol, to a final concentration of 20 units $\mu 1^{-1}$. Further dilutions were made in the same solution supplemented with $10 \%$ glycerol. Nuclease digestions were carried out with $2 \mu \mathrm{l}$ of the $1: 8000$ dilution $\left(5 \times 10^{-3}\right.$ units $)$ at $25{ }^{\circ} \mathrm{C}$ for $1 \mathrm{~min}$ and stopped with $120 \mu \mathrm{l} 40 \mathrm{mM}$ EDTA in $9 \mathrm{mM}$ Tris/HCl, pH 8.0. After phenol/chloroform purification and ethanol precipitation, samples were loaded in an ALF DNA sequencer
(Amersham Biosciences) and compared with the corresponding sequencing reaction using the same labelled primer as used for the amplification. Results were analysed with the Fragment Manager program (Amersham Biosciences).

RNA extraction and primer extension. The S. coelicolor M145 derivatives containing pIJ4083-PphoA, pIJ4083-PphoC and pIJ4083$\mathrm{P} p h o D$ were grown under the same conditions as described for the expression studies up to the time of maximum expression of each promoter (36 h for phoA and phoC; $39 \mathrm{~h}$ for phoB; see Results). RNA was then isolated by the hot phenol procedure adapted to Streptomyces (Patek et al., 2003). The transcriptional start sites were determined by primer extension using the $5^{\prime}$-fluorescein-labelled oligonucleotide O6-GCGATCGCTGCCACTGC as a primer (complementary to the $5^{\prime}$ coding region of $x y l E$ from $\mathrm{pIJ} 4083$ vector). The reaction product was loaded in an ALF DNA sequencer (as described above) and analysed with the Fragment Manager program.

\section{RESULTS}

\section{Three different S. coelicolor alkaline phosphatase genes: bioinformatic analysis indicates that PhoA, PhoC and PhoD are extracellular enzymes}

Three putative alkaline phosphatase genes, phoA, phoC and $p h o D$, were identified in the genome of $S$. coelicolor by a comparative search with the phoA gene of S. griseus (corresponding to the sequenced alkaline phosphatase protein; Moura et al., 2001). The encoded proteins PhoA (558 aa, SCO2286), PhoC (553 aa, SCO0828) and PhoD (551 aa, SCO2068) (Fig. 1) showed amino acid identities of 73, 67 and $36 \%$, respectively with S. griseus PhoA. Bioinformatic analysis of the three proteins using the PFAM and SYSTERS programs revealed that all three contain an alkaline phosphatase domain. The phoA- and phoCencoded proteins showed high similarity to the alkaline phosphatases of Bacillus species, whereas phoD encodes a protein with high similarity ( $85 \%$ identical residues) to a well-known $\mathrm{Ca}^{2+}$-dependent phospholipase D precursor of Streptomyces chromofuscus (Stieglitz et al., 2001; Yang \& Roberts, 2002). This phospholipase catalyses two reactions: (i) hydrolysis of different phospholipids to generate phosphatidic acid, and (ii) transphosphatidylation with primary or secondary alcohols to generate different phospholipids. Therefore, S. coelicolor PhoD seems to correspond to a phospholipase D (see Discussion).

Since the total phosphatase activity in S. coelicolor is a mixture of the different phosphatases, expression of each gene was quantified separately by coupling it to the $x y l E$ reporter gene.

Alkaline phosphatases and related proteins may be extracellular enzymes involved in phosphate scavenging or they may play roles in secondary metabolite biosynthesis (e.g. streptomycin-6-phosphate phosphatase encoded by strK (Mansouri \& Piepersberg, 1991) or in catabolic reactions (e.g. degradation of xenobiotics). Hydrophobicity plots using two different programs, TMpred and Tмнмм, predicted all three proteins PhoA, PhoC and PhoD to be soluble proteins. 

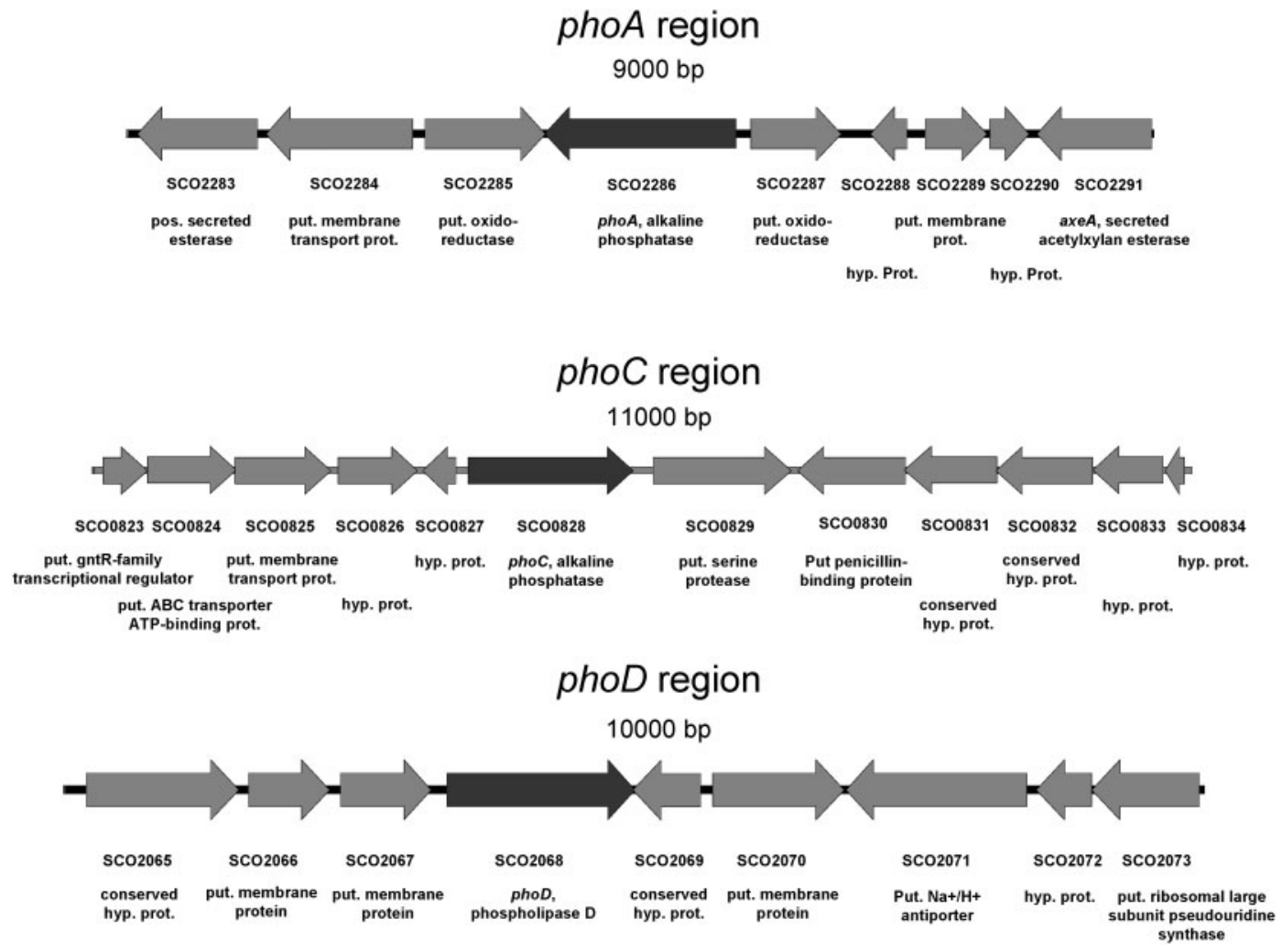

Fig. 1. Physical map of the $S$. coelicolor DNA regions around phoA, phoC and phoD (black arrows). The arrows indicate ORFs and the orientation of transcription.

PhoA (SCO2286) and PhoD (SCO2068) were shown to be secreted via the twin arginine translocation (Tat) pathway, a major route for protein secretion in Streptomyces, (Widdick et al., 2006). Their N-terminal regions contain the conserved twin-arginine motif defined as R-R-X- $\Phi-\Phi$, where $\Phi$ represents a hydrophobic amino acid (Berks, 1996). Using the TatP algorithm (Bendtsen et al., 2005) for prediction of proteins exported via the Tat pathway we identified a clear Tat motif in PhoC, with the sequence GAAARHLGRRRFLTVTAA (amino acids 23-40), nearly identical to the Tat motif RAAARSLGRRRFLTVTGA (amino acids 27-44) predicted for PhoA (Widdick et al., 2006), suggesting that PhoC might be secreted via the Tat pathway.

Using neural networks and hidden Markov models trained on Gram-positive bacteria for protein secretion via the Sec or the SRP pathway, PhoA and PhoD are also predicted to be secreted. The cleavage site of the PhoA signal peptide is predicted to lie between amino acids 62 and 63 with a probability of $95.3 \%$. The corresponding sequence is ASA/ A (/ indicates the cleavage site). The cleavage site of PhoD is located (with a probability of $81.3 \%$ ) between amino acids 44 and 45 in the sequence ADA/A. The overall probability for both proteins to be secreted is $95.5 \%$ for PhoA and $100 \%$ for PhoD. The above-mentioned models, which are trained to predict proteins secreted via the Sec or the SRP pathway, indicate that PhoC is not secreted through these secretion pathways (with a secretion probability of only $30.5 \%$ ).

\section{Expression of phoA is strongly induced by phosphate limitation and this induction is PhoP-dependent}

The promoter of phoA (PphoA) in S. coelicolor was weakly expressed in high phosphate but highly expressed in lowphosphate conditions (Fig. 2a). In phosphate-limited batch cultures phoA is efficiently expressed, as measured by quantification of reporter activity, and reached its maximum activity at about $39 \mathrm{~h}$ (Fig. 2a), decreasing thereafter.

Expression of phoA was nearly non-existent in the $S$. coelicolor $\triangle p h o P$ mutant, indicating that $\mathrm{PhoP}$ is strictly required for expression of this promoter, i.e. phoA expression is dependent upon activation by PhoP.

\section{The promoter of phoC shows a weak transcriptional activity and is not PhoP-dependent}

Expression of phoC in wild-type S. coelicolor M145 is much lower than that of phoA measured using the same reporter system. The profile of expression of phoC was clearly 
(a)

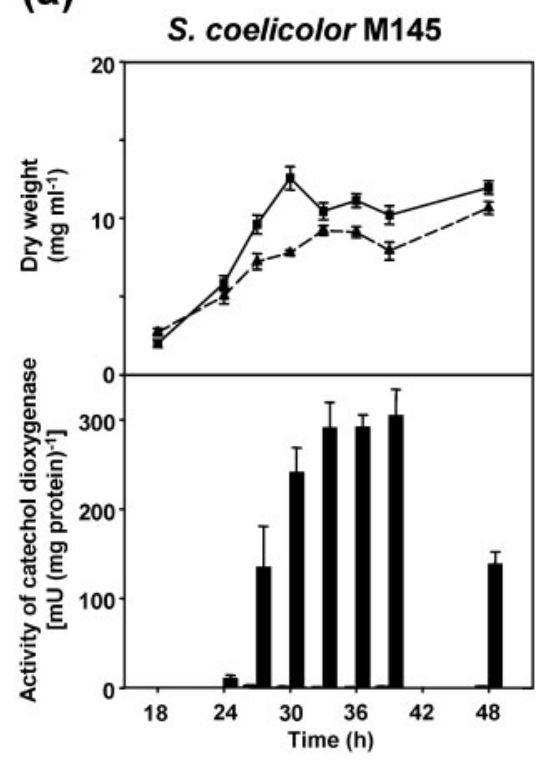

S. coelicolor $\Delta$ phoP

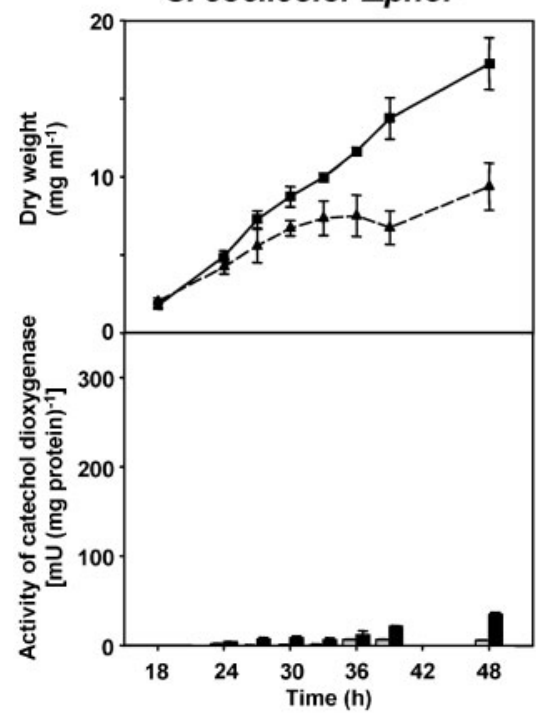

(b)

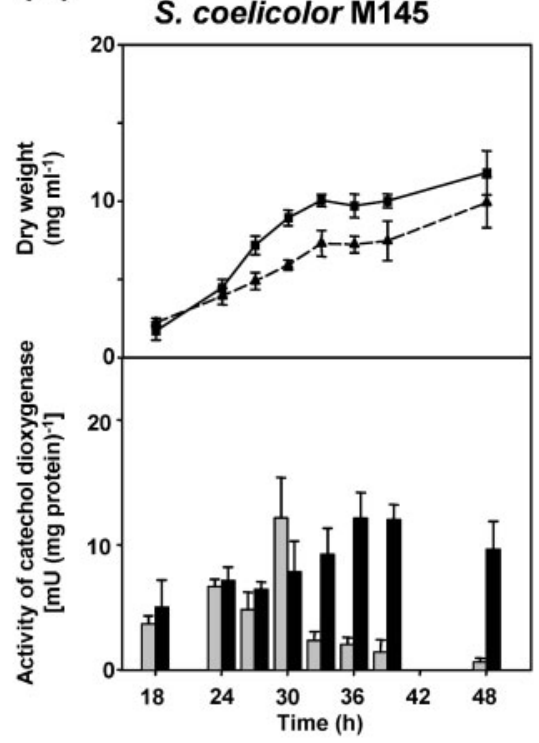

S. coelicolor $\Delta p h o P$

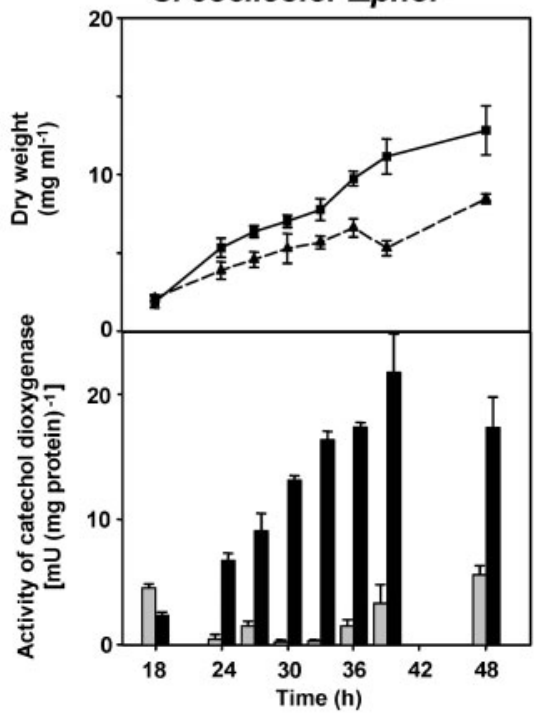

(c)

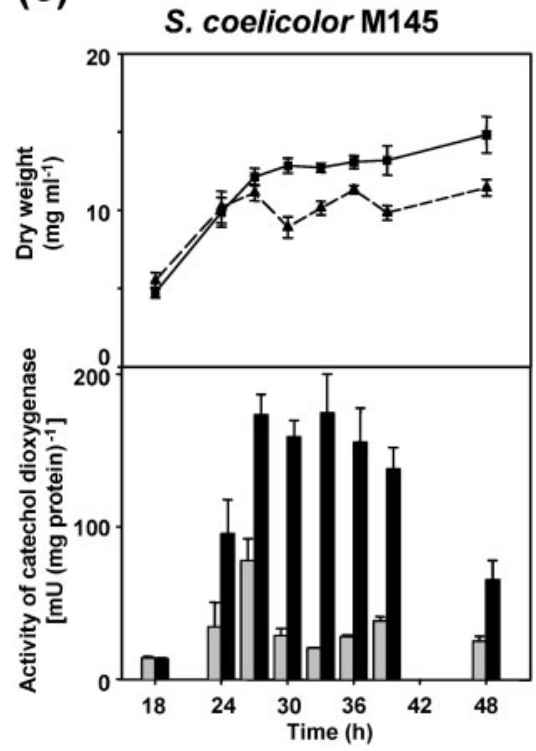

S. coelicolor $\Delta$ phoP

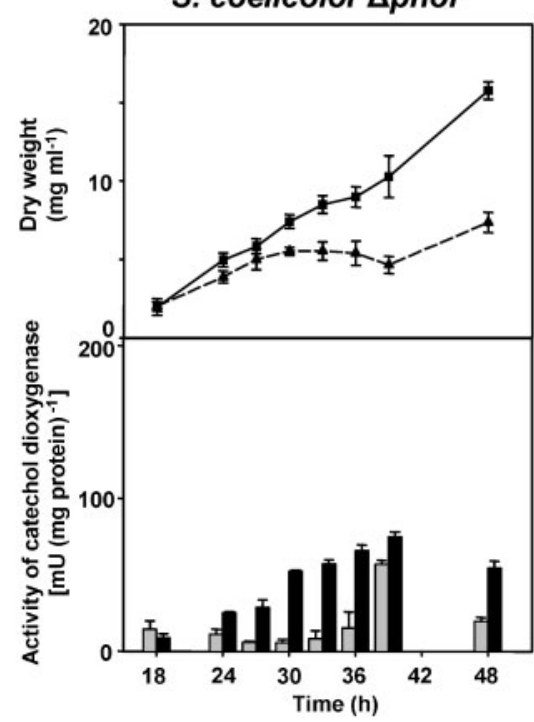

Fig. 2. Phosphate control of expression of $p h o A(a)$, phoC (b) and phoD (c) in the parental strain $S$. coelicolor $M 145$ and the $\triangle p h o P$ mutant (upper and lower panels, respectively), using the promoters of each of the three genes coupled to the $x y / E$ reporter gene. Cultures of each strain were performed in R5 medium supplemented with $0.009 \mathrm{mM}$ phosphate ( $\boldsymbol{\Lambda}$ and black bars) or with $1.8 \mathrm{mM}$ phosphate ( $\boldsymbol{\square}$ and grey bars). The growth curves of each transformant are shown in the upper part of each panel. The error bars indicate SD $(n=6)$. Note the different scales for XyIE (catechol dioxygenase) activity for the three promoters.

different from that of phoA. It was induced under lowphosphate concentrations, activity reaching its maximum after 36-39 h in R5 phosphate-limited medium, and not decreasing significantly until after $48 \mathrm{~h}$ of growth (Fig. 2b). However, its expression was not maintained after $30 \mathrm{~h}$ in phosphate-supplemented medium, indicating that this gene might be subject to a complex dual regulation by phosphate. This gene does not encode a typical alkaline phosphatase (see Discussion) and its expression may be modulated differently in low- and high-phosphate conditions.
The expression of phoC is negatively regulated by phosphate in both the wild-type and the $\Delta$ phoP mutant. In conditions of phosphate sufficiency the expression of phoC is only active in early growth (up to $30 \mathrm{~h}$ ) then abruptly decreases. This early expression of $p h o C$ is not observed in the phoP mutant. A similar observation was made in the case of $p h o D$. In order to explain these observations, we can argue that $p h o C$ and $p h o D$ are expressed during the exponential growth phase in highphosphate conditions by a transcription factor that 
depends on the presence of PhoP. The later expression of phoC observed under phosphate-limited conditions is not under the positive control of $\mathrm{PhoP}$, since its expression is even higher in the phoP mutant strain; this indicates the existence of an unknown phosphate-responsive regulator, probably detecting intracellular phosphate limitation, which is more severe in the case of the phoP mutant due to its inability to activate the expression of genes of the $\mathrm{PHO}$ regulon.

\section{Expression of phoD is highly regulated by phosphate and partially PhoP-dependent}

The expression of phoD increased under low-phosphate conditions, reaching an expression level nearly as high as that of $p h o A$. Its expression was also significantly reduced by $1.8 \mathrm{mM}$ phosphate.

In the $\Delta p h o P$ mutant the overall $p h o D$ expression decreased significantly (but was not completely suppressed) when compared to wild-type strain M145, indicating that although PhoP is clearly involved in the phosphate control of phoD, its expression is not as strictly PhoP-dependent, as observed for phoA (Fig. 2c). In conditions of phosphate limitation, the expression of $p h o D$ is very active throughout growth and this expression is clearly dependent upon PhoP. In conditions of phosphate sufficiency, the expression of $p h o D$ is active in early growth then abruptly decreases as for $p h o C$; this early expression is not detected in the $\Delta p h o P$ mutant whereas a late expression $(40 \mathrm{~h})$ is observed. Our results suggest that another regulatory element besides PhoP plays a role in phoD induction under conditions of phosphate limitation.

\section{Characterization of the promoters by primer extension}

The transcription start points of the phoA, phoC and phoD genes were determined by primer extension. In all three cases the origins of transcription were identified and, once the +1 sites were known, the corresponding -10 and -35 boxes of each promoter were established by comparison to the matrices reported by Bourn \& Babb (1995) for Streptomyces that take into account the nucleotides occurring in 13-nucleotide stretches, including the -10 or -35 consensus hexamers, as described by Sola-Landa et al. (2005).

Two different transcription start points (tsp1 and tsp2) were found upstream of the phoA open reading frame (Fig. 3). The first and stronger one was located at a guanosine $25 \mathrm{bp}$ upstream of the start codon and corresponds to a -10 box (TCGAAT) and -35 box (CAAACC) separated by $18 \mathrm{bp}$. For the second transcription start point, located $53 \mathrm{bp}$ upstream of the start codon, no consensus -10 and -35 boxes could be identified, suggesting that this second transcription start point is probably recognized by a different sigma factor. A putative RBS with the consensus core sequence GGAGG was found

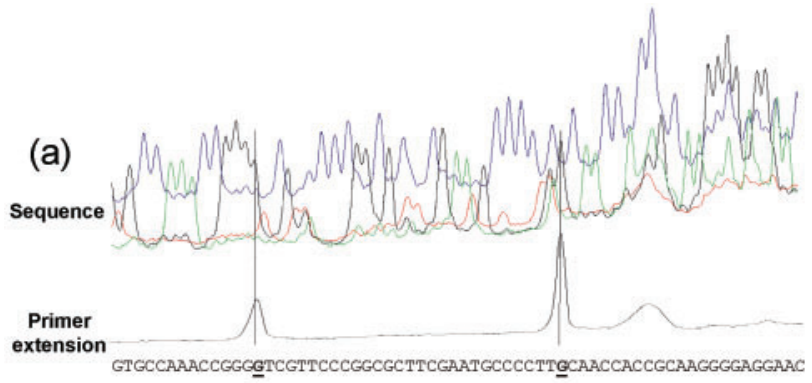

(b)

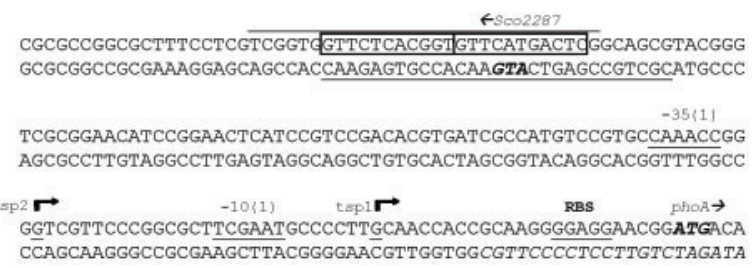

Fig. 3. Primer extension analysis of the phoA promoter (a) and nucleotide sequence of its promoter region (b). Two peaks were found in the primer extension analysis using $50 \mu \mathrm{g}$ RNA. The start codon of $p h o A$ and of its upstream gene are indicated in bold. The transcription start points tsp1 and tsp 2 are indicated by bent arrows; the -10 and -35 region and the putative RBS are underlined. The region protected by PhoP in DNase footprinting assays is over- and underlined in the corresponding strand. The $\mathrm{PHO}$ sequence is indicated by boxes.

$6 \mathrm{bp}$ upstream of the ATG translation start initiation codon of phoA.

Transcription of phoC initiates from two different transcription start points (tsp1 and tsp2) located $25 \mathrm{bp}$ and $50 \mathrm{bp}$ upstream of the start codon (Fig. 4). For both sites possible -10 and -35 boxes with the sequences -10 TCCGCG/-35 TTTTGT (tsp1) and - 10 CAGGGC/-35 ATCACT (tsp2) separated by $17 \mathrm{bp}$ (tsp1) and $18 \mathrm{bp}$ (tsp2) could be identified. A possible RBS with the core sequence AGAGG is located $7 \mathrm{bp}$ upstream of the start codon of phoC.

Transcription of phoD initiates at the first guanosine of its GTG translation start codon, for which no RBS was identified. A second GTG codon is found $69 \mathrm{bp}$ downstream, but comparison of PhoD with the homologous proteins from other Streptomyces sequences shows high conservation within the first 23 amino acids and as those include the Tat secretion signal predicted by Widdick et al. (2006), translation is likely to start at the first GTG (Fig. 5). Its -10 box (TAACCT) and -35 box (CCCACT) are separated by $18 \mathrm{bp}$ and overlap with the second protected region and the PHO box repeats located in it (Fig. 5).

All transcription initiation points were consistent with the identified -10 and -35 regions. We cannot exclude, however, other minor transcription start points that do not show up when using the primer extension technique. 


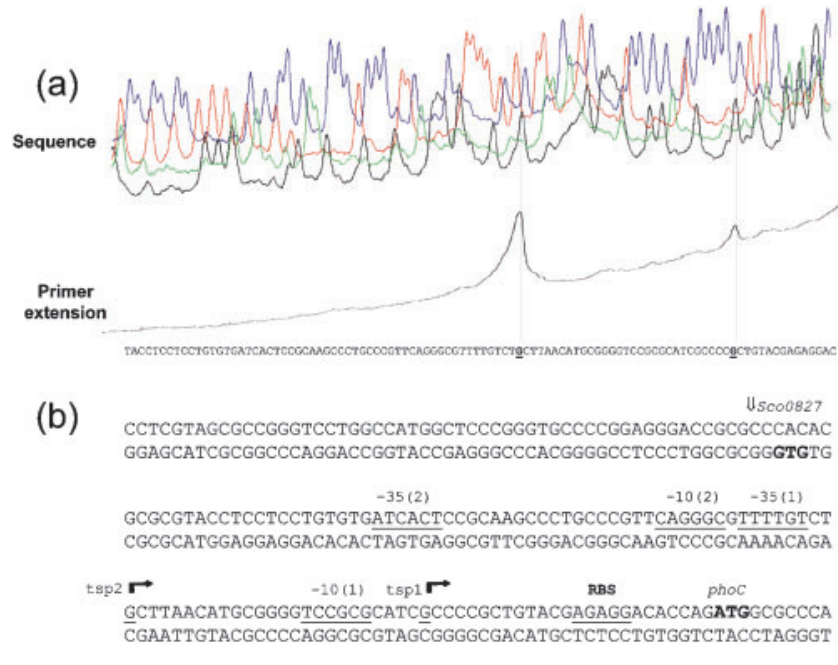

Fig. 4. Primer extension analysis of the phoC promoter (a) and nucleotide sequence of its promoter region (b). Two peaks were found in the primer extension analysis, which was carried out with $200 \mu \mathrm{g}$ RNA. The start codon of phoC and of its upstream gene are indicated in bold. The transcription start points are shown by bent arrows; the -10 and -35 region and the putative RBS are underlined. No consensus $\mathrm{PHO}$ boxes are evident in this promoter.

Upstream of the phoD gene there are three ORFs transcribed in the same orientation (Fig. 1); one of them encodes a conserved hypothetical protein (SCO2065) and the other two encode putative membrane proteins (SCO2066/SCO2067). Downstream of phoD there is a conserved hypothetical protein arranged in the opposite orientation. In the expression studies we observed promoter activity for the DNA region upstream of phoD (Fig. 2). The possibility that phoD is additionally transcribed in an operon together with these three genes seems unlikely because of the $14 \mathrm{bp}$ palindromic sequence located between the transcription start point of phoD and SCO2067 (Fig. 5) that could form a stable stem-loop structure in the RNA and function as a transcriptional terminator.

\section{PhoP binds to the promoters of phoA and phoD, but not to that of phoC}

The interaction of PhoP with the three promoters was tested by EMSAs using the S. coelicolor GST-PhoP fusion protein as well as a truncated version of PhoP containing only its DNA-binding domain (C-terminal region; GST$\mathrm{PhoP}^{\mathrm{DBD}}$ ). The $\mathrm{PhoP}^{\mathrm{DBD}}$ fragment shows constitutive binding to $\mathrm{PHO}$ boxes, independent of its phosphorylation status (Sola-Landa et al., 2005).

The promoters of phoA (Fig. 6a) and phoD (Fig. 6c) showed a clear shift of the labelled DNA band. In both cases the binding was stronger with the truncated PhoP ${ }^{\mathrm{DBD}}$ than with the complete PhoP protein. In the EMSAs performed with the $p h o D$ promoter up to four bands were
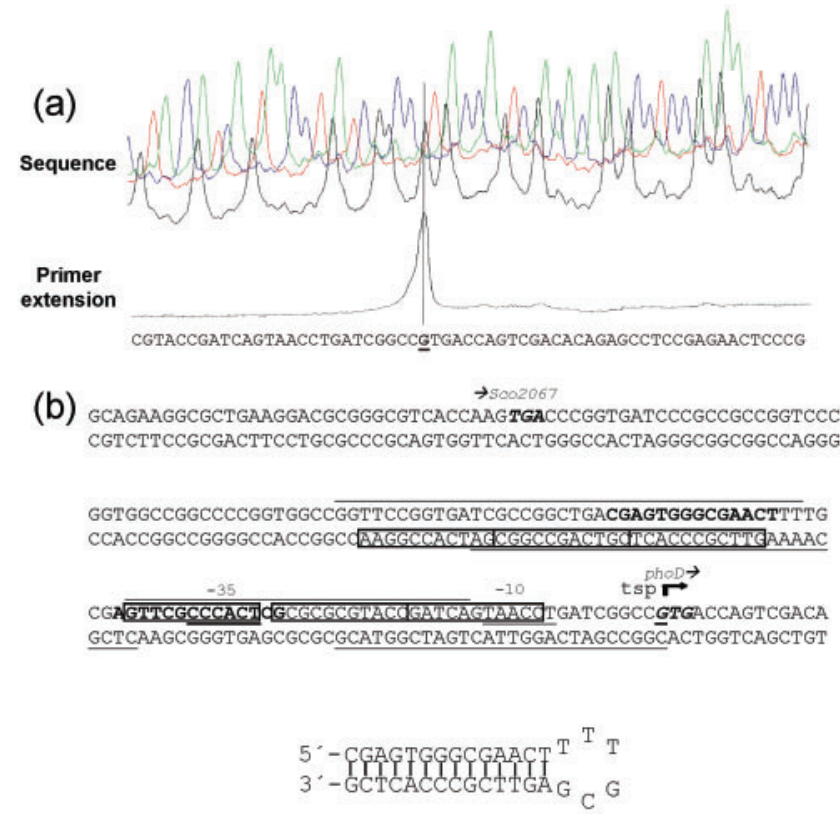

Fig. 5. Primer extension analysis of the phoD promoter (a) and nucleotide sequence of its promoter region (b). One clear transcription start point could be identified by primer extension carried out with $50 \mu \mathrm{g}$ RNA. The start codon of $p h o D$ and the stop codon of its upstream gene are indicated in bold. The transcription start point is indicated by a bent arrow; the -10 and -35 regions are underlined. The region protected by PhoP in DNase footprinting assays is over- and underlined in the corresponding strand; $\mathrm{PHO}$-sequences are indicated by boxes. A 14 bp palindromic sequence, probably working as a transcriptional terminator for the upstream gene, is indicated in bold. The stem-loop structure of the putative terminator is shown below the promoter sequence.

observed, indicating that several DNA-protein complexes were formed due to binding of increasing amounts of Pho ${ }^{\mathrm{DBD}}$ to the three Pho boxes existing in this promoter (see below), whereas the promoter of phoA formed only one shifted band.

An interesting finding was that the promoter of phoC did not show a shift reaction with either the complete PhoP or the truncated PhoP ${ }^{\mathrm{DBD}}$ version (Fig. 6b) under the same conditions as used for EMSA that showed mobility shifts for the $p h o A$ and $p h o D$ promoters. These results agree with the expression studies (Fig. 2b) and suggest that the phoC promoter lacks adequate $\mathrm{PHO}$ boxes. Computer analysis of the phoC promoter confirmed the absence of PHO boxes in this promoter, supporting the observed lack of binding of the PhoP response regulator to this DNA region.

\section{Footprinting assays of $p h o A$ and $p h o D$ : identification of PHO boxes in these promoters}

To locate the PHO box repeat units in the promoters of phoA and phoD, footprinting assays were performed using 

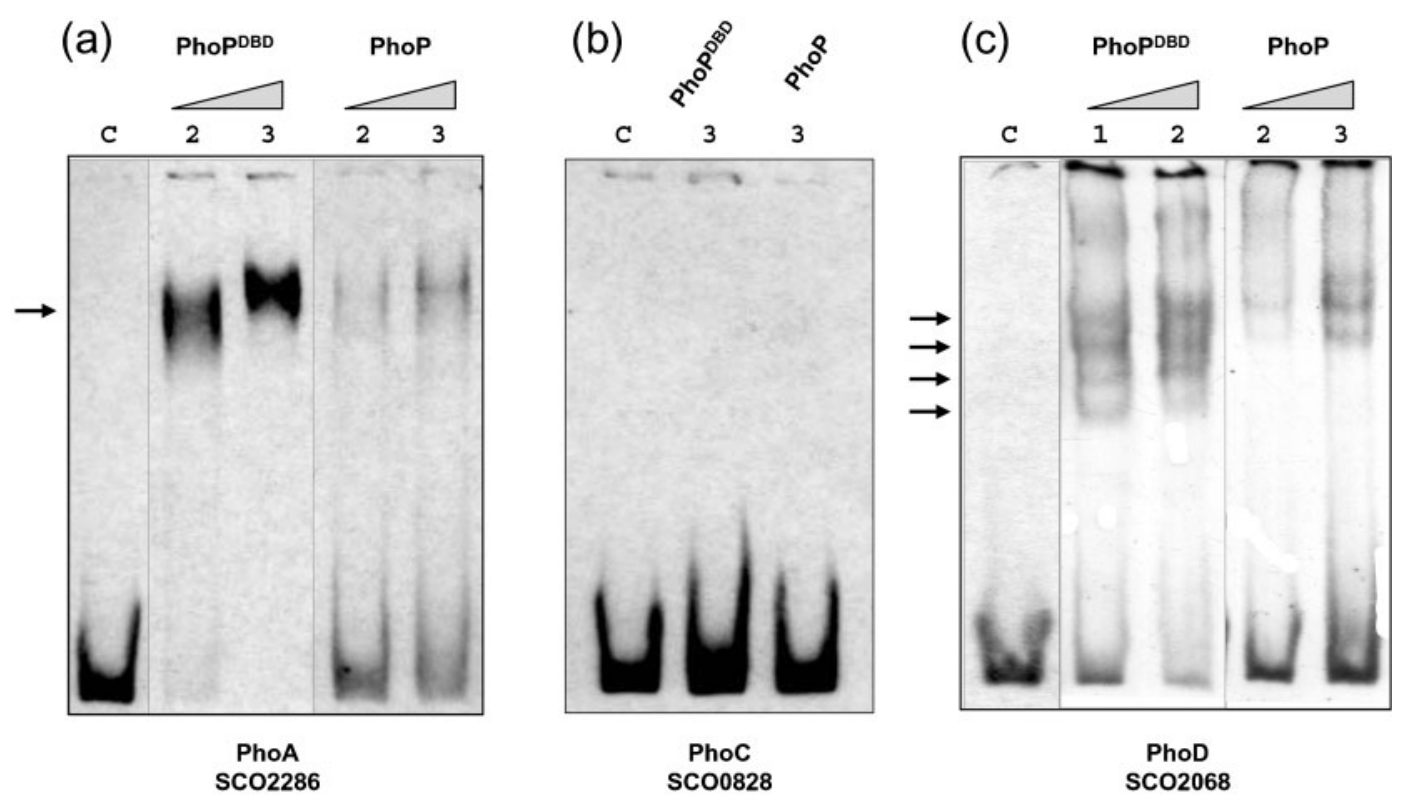

Fig. 6. EMSAs of the promoter regions of $p h o A(a)$, phoC (b) and phoD (c) using different concentrations of GST-PhoP ${ }^{\mathrm{DBD}}$ and GST-PhoP. Lanes: C, Control without protein; 1, 6.25 pmol; 2, 12.5 pmol; 3, 25 pmol protein. Arrows indicate shifted bands.

the PhoP ${ }^{\mathrm{DBD}}$ protein to protect them against DNAse I digestion.

Results of the footprinting studies identified in the phoA promoter a PhoP-protected region of $29 \mathrm{bp}$ in the coding strand and of $29 \mathrm{bp}$ in the complementary strand, overlapping by $23 \mathrm{bp}$. In this region one complete Pho box containing two repeats of $11 \mathrm{bp}$ (Sola-Landa et al., 2005) with the sequence GTTCTCACGGT GTTCATGACTC was identified (the first nucleotide of each repeat is underlined) (Fig. 7). This sequence is located in the coding strand 103 or 75 bp upstream of the experimentally determined transcription start points tsp1 and tsp2, respectively (see below).

In the phoD promoter two protected regions of $38 \mathrm{bp}$ and 28 bp (separated by $6 \mathrm{bp}$ ) in the coding strand and $33 \mathrm{bp}$ and $27 \mathrm{bp}$ (separated by $16 \mathrm{bp}$ ) in the complementary strand were identified, overlapping by $27 \mathrm{bp}$ and $11 \mathrm{bp}$, respectively (Fig. 8).

The upstream protected region contains three direct $11 \mathrm{nt}$ repeats located in the complementary strand with the sequence GTTCGCCCACT CGTCAGCCGGC GATCACCGGAA (the first nucleotide of each repeat is underlined). The downstream protected region contains three direct repeats with the sequence GTTCGCCCACT C GCGCGCGTACC GATCAGTAACC located in the coding strand and separated by one nucleotide $(\mathrm{C})$ between the first and the following two repeats (Fig. 8) (see Discussion).

The Pho box repeats in phoD are located $10 \mathrm{bp}$ from the experimentally determined transcription start point, overlapping with the predicted -10 and -35 boxes (see below).

\section{DISCUSSION}

Three different alkaline phosphatase genes were initially described in the S. coelicolor genome (Moura et al., 2001; Bentley et al., 2002). Interestingly, similar BLAST searches in the genomes of Streptomyces avermitilis and Streptomyces scabies only identified two possible alkaline phosphatases, corresponding to $p h o A$ and $p h o D$; so the phoC gene of $S$. coelicolor seems to be a more recent addition to the $S$. coelicolor genome and might be involved in the biosynthesis of some S. coelicolor-specific metabolite. PhoA of $S$. coelicolor corresponds to an authentic alkaline phosphatase with high similarity to the well-known extracellular alkaline phosphatase of S. griseus (Moura et al., 2001) and other micro-organisms. It has been shown to be secreted via the Tat pathway (Widdick et al., 2006) but also contains a typical leader peptide as described for Secdependent secretion (Schaerlaekens et al., 2004).

Expression of phoA is strongly activated by phosphate limitation (Fig. 2a) and is strictly dependent upon the binding of PhoP to the consensus PHO box identified in its promoter (see below). Transcriptional analysis revealed the existence of two transcription start sites, tsp1 and tsp2, allowing the definition of two promoters. DNAse I footprinting experiments revealed the presence of putative PHO boxes (two direct repeats of $11 \mathrm{bp}$ ) located 103 and $75 \mathrm{bp}$ upstream of tsp1 and tsp2 respectively. These $\mathrm{PHO}$ boxes are in the 

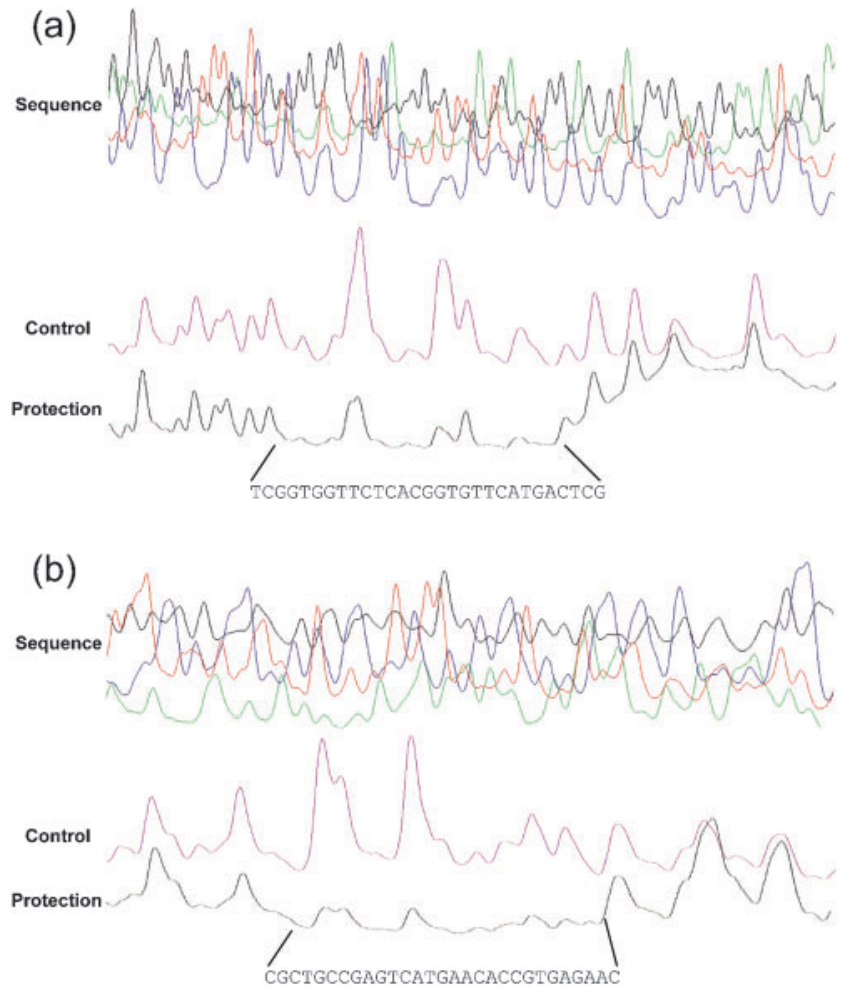

Fig. 7. Footprinting of the coding strand (a) and the complementary strand (b) of the phoA promoter region using GST-PhoP ${ }^{\mathrm{DBD}}$. Shown is the sequencing reaction (above), a control without protein (middle) and the footprinting (below) using $1.06 \mu \mathrm{M}$ GST$\mathrm{PhoP}{ }^{\mathrm{DBD}}$. The sequence of the protected region is given.

position of an activator site for both promoters that are thus both likely to be regulated by phosphate availability.

phoC encodes an alkaline phosphatase similar to PhoA, but its regulation pattern is clearly different from that of phoA and $p h o D$. Its expression can be divided into two phases: an early PhoP-dependent phase up to $30 \mathrm{~h}$ and a later phase not directly dependent upon PhoP. Under conditions of phosphate limitation, the expression of $p h o C$ is active in early growth stages but keeps on increasing during later growth and is even higher in the phoP mutant.

Although the TatP program (Bendtsen et al., 2005) predicts PhoC to be secreted via the Tat pathway, the KEGG database, a knowledge base for systematic analysis of gene functions (Kanehisa et al., 2006), classifies PhoC as a phosphoric monoester hydrolase that may be involved in two pathways: (i) $\gamma$-hexachlorocyclohexane degradation and (ii) folate biosynthesis. Although both pathways occur in S. coelicolor (PATHS SCO00361 and SCO00790, respectively) there is no unequivocal evidence to conclude that PhoC corresponds to the phosphatase catalysing the dephosphorylation steps of these pathways.

The lack of binding of the PhoP response regulator to the phoC promoter suggests that the observed phosphate regulation of the phoC gene is not mediated by direct interaction of $\mathrm{PhoP}$, in agreement with the lack of $\mathrm{PHO}$ boxes in this promoter region. Phosphate regulation of phoC during the early growth phase might involve an intermediary regulator whose expression might be under the control of PhoR/PhoP. The phosphate-response regulator PhoP binds to a significant number of phosphate-regulated genes in Streptomyces (Sola-Landa et al., 2005), but other phosphate-controlled genes lack Pho boxes and are not recognized by PhoP (Rodríguez-García et al., 2007).

The S. coelicolor phoD gene corresponds to a novel-type $\mathrm{Ca}^{2+}$-dependent phospholipase similar to that of $S$. chromofuscus ( $85 \%$ identical amino acid residues). These phospholipases of S. coelicolor and S. chromofuscus show little homology with other members of the phospholipase D superfamily. This PhoD phospholipase is also different from classical phospholipases described in other Streptomyces species. The PhoD proteins of $S$. coelicolor and $S$. chromofuscus lack the identifiable HKD motif common to members of the phospholipase D family. The S. chromofuscus enzyme has been characterized biochemically and shows phosphodiesterase activity; it is secreted and cleaved by an extracellular protease, increasing its enzymic activity (Geng et al., 1999; Yang \& Roberts, 2002). This enzyme binds to membrane phosphatidylcholine and facilitates vesicle aggregation and fusion (Stieglitz et al., 1999, 2001).

Transcriptional analysis revealed the existence of a unique transcriptional start point coinciding with the first base of the translation initiation codon, allowing the definition of an unique promoter. The expression of phoD is activated under phosphate-limited conditions by PhoP, which binds to the two regions of $11 \mathrm{bp}$ repeat units ( $\mathrm{PHO}$ box) found in its promoter, overlapping partially with its predicted -10 and -35 boxes. The formation of up to four DNA-PhoP bands in the EMSA experiments correlates with the distinct organization of the PHO boxes in the promoter of this gene. The activation of phoD in the mutant strain S. coelicolor $\Delta p h o P($ INB101) is weaker than that in the wild-type strain. This activation in the $\Delta p h o P$ mutant under phosphatelimitation conditions suggests that another regulatory factor besides PhoP plays a role in phosphate control.

The sequence of PHO boxes protected by binding of the PhoP protein was established recently in the promoters of the pstS, phoRP and phoU genes (Sola-Landa et al., 2005; Rodríguez-García et al., 2007). However, the number of validated PHO boxes in Streptomyces genomes is still very limited. The presence of two $11 \mathrm{bp}$ direct repeats in the phoA promoter and six repeats in the $p h o D$ promoter that match the protected region allowed us to establish a new, better-fitting sequence for the Pho box as $\mathrm{G}(82 \%) \mathrm{G} /$ $\mathrm{T}(85 \%) \mathrm{T}(77 \%) \mathrm{C}(90 \%) \mathrm{A}(59 \%) \mathrm{Y}(82 \%) \mathrm{Y}(74 \%) \mathrm{Y}(72 \%)$ $\mathrm{R}(62 \%) \mathrm{Y}(64 \%) \mathrm{Y}(74 \%)$.

Duplication of the number of PHO boxes in certain promoters is related to a potential modulation of the strong phosphate control (Mendes et al., 2007). The 

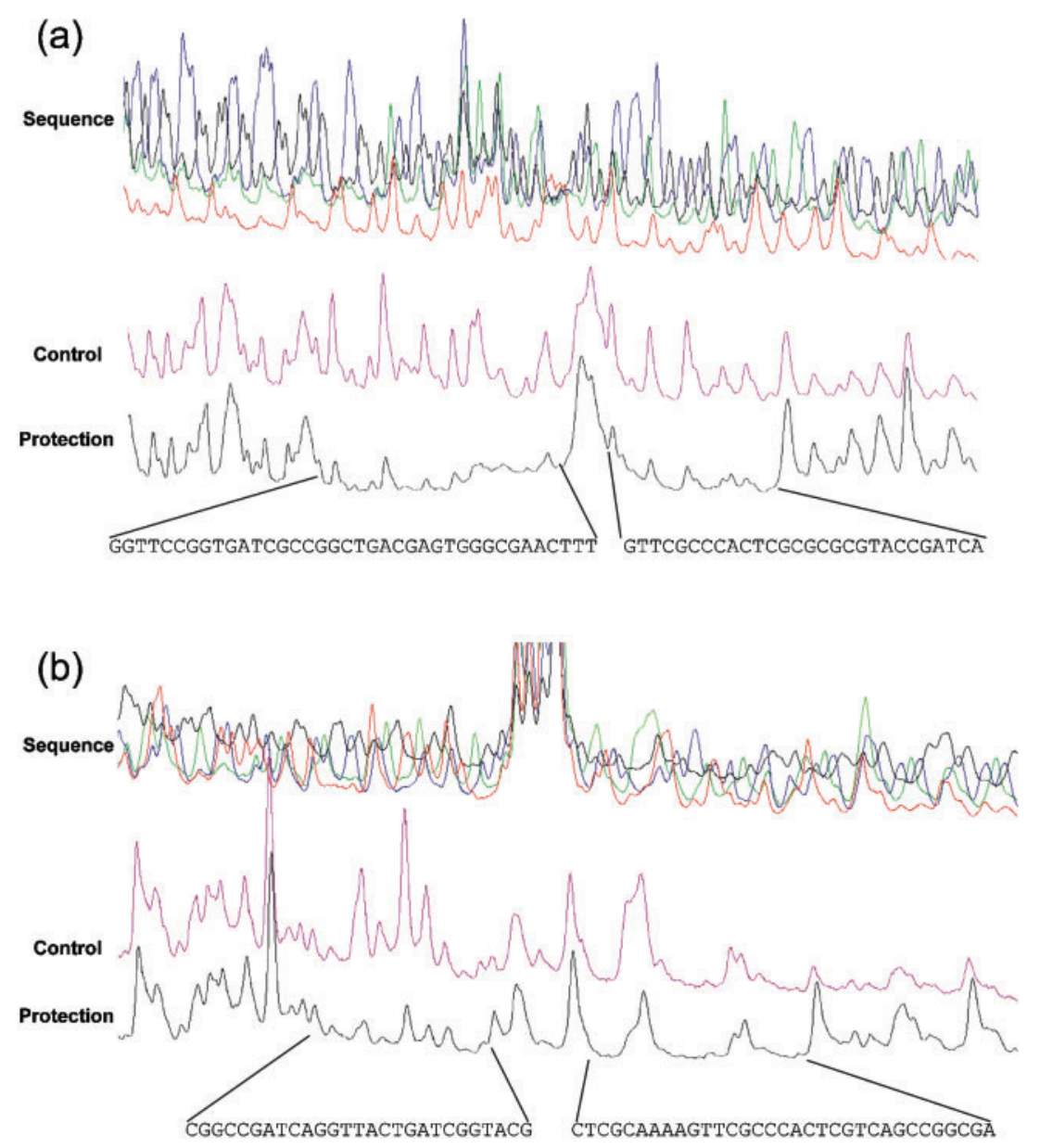

Fig. 8. Footprinting of the coding strand (a) and the complementary strand (b) of the phoD promoter region using GST-PhoP ${ }^{\mathrm{DBD}}$. Shown is the sequencing reaction (above), a control without protein (middle) and the footprinting (below) using $1.06 \mu \mathrm{M}$ GST-PhoP ${ }^{\mathrm{DBD}}$. The sequence of the protected region is given.

formation of up to four DNA-PhoP protein complexes in the binding reaction of the phoD promoter suggests that this promoter is subject to different degrees of PhoP control depending on the level of the phosphorylated response regulator in the cell.

\section{ACKNOWLEDGEMENTS}

This work was supported by grants of the CICYT (BIO2003-01489), Madrid (Spain) and the European Union (Project ACTINOGEN, LSHM-CT-2004-005224) Brussels. A. K. Apel received a F.P.U. fellowship of the Ministry of Education and Science. We thank J. Merino, B. Martín, B. Aguado and A. Casenave for their excellent technical assistance.

\section{REFERENCES}

Bendtsen, J. D., Nielsen, H., Widdick, D., Palmer, T. \& Brunak, S. (2005). Prediction of twin-arginine signal peptides. BMC Bioinformatics 6, 167.

Bentley, S. D., Chater, K. F., Cerdeno-Tarraga, A. M., Challis, G. L., Thomson, N. R., James, K. D., Harris, D. E., Quail, M. A., Kieser, H. \& other authors (2002). Complete genome sequence of the model actinomycete Streptomyces coelicolor A3(2). Nature 417, 141-147.

Berks, B. C. (1996). A common export pathway for proteins binding complex redox cofactors? Mol Microbiol 22, 393-404.
Bourn, W. R. \& Babb, B. (1995). Computer assisted identification and classification of streptomycete promoters. Nucleic Acids Res 23, 3696-3703.

Clayton, T. M. \& Bibb, M. J. (1990). Streptomyces promoter-probe plasmids that utilise the xylE gene of Pseudomonas putida. Nucleic Acids Res 18, 1077.

Demain, A. L. \& Fang, A. (2000). The natural functions of secondary metabolites. Adv Biochem Eng Biotechnol 69, 1-39.

Fernández-Ábalos, J. M., Sánchez, P., Coll, P. M., Villanueva, J. R., Pérez, P. \& Santamaría, R. I. (1992). Cloning and nucleotide sequence of celA1, and endo- $\beta$-1,4-glucanase-encoding gene from Streptomyces halstedii JM8. J Bacteriol 174, 6368-6376.

García-González, M. D., Martín, J. F., Vigal, T. \& Liras, P. (1991). Characterization, expression in Streptomyces lividans, and processing of the amylase of Streptomyces griseus IMRU 3570: two different amylases are derived from the same gene by an intracellular processing mechanism. J Bacteriol 173, 2451-2458.

Geng, D., Baker, D. P., Foley, S. F., Zhou, C., Stieglitz, K. \& Roberts, M. F. (1999). A $20-\mathrm{kDa}$ domain is required for phosphatidic acidinduced allosteric activation of phospholipase D from Streptomyces chromofuscus. Biochim Biophys Acta 1430, 234-244.

Ghorbel, S., Kormanec, J., Artus, A. \& Virolle, M. J. (2006). Transcriptional studies and regulatory interactions between the phoRphoP operon and the phoU, mtpA, and ppk genes of Streptomyces lividans TK24. J Bacteriol 188, 677-686.

Hanahan, D. (1983). Studies on transformation of Escherichia coli with plasmids. J Mol Biol 166, 557-580. 
Kanehisa, M., Goto, S., Hattori, M., Aoki-Kinoshita, K. F., Itoh, M., Kawashima, S., Katayama, T., Araki, M. \& Hirakawa, M. (2006). From genomics to chemical genomics: new developments in KEGG. Nucleic Acids Res 34, D354-D357.

Kieser, T., Bibb, M., Buttner, M. J., Chater, K. F. \& Hopwood, D. A. (2000). Practical Streptomyces Genetics. Norwich, UK: The John Innes Foundation.

Mansouri, K. \& Piepersberg, W. (1991). Genetics of streptomycin production in Streptomyces griseus: nucleotide sequence of five genes, strFGHIK, including a phosphatase gene. Mol Gen Genet 228, 459-469.

Martín, J. F. (2004). Phosphate control of the biosynthesis of antibiotics and other secondary metabolites is mediated by the PhoR-PhoP system: an unfinished story. J Bacteriol 186, 5197-5201.

Martín, J. F. \& Demain, A. L. (1977). Cleavage of adenosine-5' monophosphate during uptake by Streptomyces griseus. J Bacteriol 132, 590-595.

Martín, J. F. \& Demain, A. L. (1980). Control of antibiotic synthesis. Microbiol Rev 44, 230-251.

Martín, J. F., Marcos, A. T., Martín, A., Asturias, J. A. \& Liras, P. (1994). Phosphate control of antibiotic biosynthesis at the transcriptional level. In Phosphate in Microorganisms: Cellular and Molecular Biology, pp. 140-147. Edited by A. Torriani-Gorini, E. Yagil \& S. Silver. Washington, DC: American Society for Microbiology.

Martín, J. F., Gutiérrez, S. \& Aparicio, J. F. (2000). Secondary metabolites. In Encyclopedia of Microbiology, vol. 4, 2nd edn, pp. 213 236. Edited by J. Lederberg. San Diego, CA: Academic Press.

Martínez-Domínguez, B., Ibáñez-Gómez, M. V. \& Rincón-León, F. (2002). Phytic acid: nutritional aspects and analytical implications. Arch Latinoam Nutr 52, 219-231.

Mendes, M. V., Tunca, S., Antón, N., Recio, E., Sola-Landa, A., Aparicio, J. F. \& Martín, J. F. (2007). The two-component phoR-phoP system of Streptomyces natalensis: inactivation or deletion of phoP reduces the negative phosphate regulation of pimaricin biosynthesis. Metab Eng 9, 217-227.

Moura, R. S., Martín, J. F., Martín, A. \& Liras, P. (2001). Substrate analysis and molecular cloning of the extracellular alkaline phosphatase of Streptomyces griseus. Microbiology 147, 1525-1533.

Patek, M., Muth, G. \& Wohlleben, W. (2003). Function of Corynebacterium glutamicum promoters in Escherichia coli, Streptomyces lividans, and Bacillus subtilis. J Biotechnol 104, 325-334.
Rodríguez-García, A., Ludovice, M., Martín, J. F. \& Liras, P. (1997). Arginine boxes and the $\arg \mathrm{R}$ gene in Streptomyces clavuligerus: evidence for a clear regulation of the arginine pathway. Mol Microbiol 25, 219-228.

Rodríguez-Garcia, A., Barreiro, C., Santos-Beneit, F., Sola-Landa, A. \& Martín, J. F. (2007). Genome-wide transcriptomic and proteomic analysis of the primary response to phosphate limitation in Streptomyces coelicolor M145 and in a $\Delta$ phoP mutant. Proteomics 7 , 2410-2429.

Schaerlaekens, K., Lammertyn, E., Geukens, N., De Keersmaeker, S., Anne, J. \& Van Mellaert, L. (2004). Comparison of the Sec and Tat secretion pathways for heterologous protein production by Streptomyces lividans. J Biotechnol 112, 279-288.

Sola-Landa, A., Moura, R. S. \& Martín, J. F. (2003). The twocomponent PhoR-PhoP system controls both primary metabolism and secondary metabolite biosynthesis in Streptomyces lividans. Proc Natl Acad Sci U S A 100, 6133-6138.

Sola-Landa, A., Rodríguez-García, A., Franco-Domínguez, E. \& Martín, J. F. (2005). Binding of PhoP to promoters of phosphate regulated genes in Streptomyces coelicolor: identification of $\mathrm{PHO}$ boxes. Mol Microbiol 56, 1373-1385.

Stieglitz, K., Seaton, B. \& Roberts, M. F. (1999). The role of interfacial binding in the activation of Streptomyces chromofuscus phospholipase D by phosphatidic acid. J Biol Chem 274, 35367-35374.

Stieglitz, K. A., Seaton, B. A. \& Roberts, M. F. (2001). Binding of proteolytically processed phospholipase D from Streptomyces chromofuscus to phosphatidylcholine membranes facilitates vesicle aggregation and fusion. Biochemistry 40, 13954-13963.

von Döhren, H. \& Gräfe, H. (1997). General aspects of secondary metabolism. In Biotechnology, vol. 7. Products of Secondary Metabolism, pp. 1-55. Edited by H. J. Rehm \& G. Reed. Weinheim: VCH Verlag.

Widdick, D. A., Dilks, K., Chandra, G., Bottrill, A., Naldrett, M., Pohlschröder, M. \& Palmer, T. (2006). The twin-arginine translocation pathway is a major route of protein export in Streptomyces coelicolor. Proc Natl Acad Sci U S A 103, 17927-17932.

Yang, H. \& Roberts, M. F. (2002). Cloning, overexpression, and characterization of a bacterial $\mathrm{Ca}^{2+}$-dependent phospholipase D. Protein Sci 11, 2958-2968.

Edited by: J. Anné 